\title{
Orthogonal Self-Assembly of Carbon Nanotube Crossbar Architectures by Simultaneous Graphoepitaxy and Field-Directed Growth
}

Ariel Ismach and Ernesto Joselevich*

Department of Materials and Interfaces, Weizmann Institute of Science, Rehovot 76100, Israel.

E-mail: ernesto.joselevich@weizmann.ac.il

\section{Supporting Information}

\section{Definition of the miscut parameters}

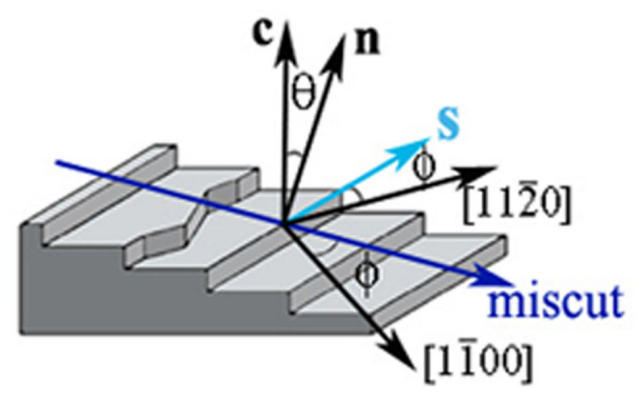

Figure S1. Miscut inclination and azimuth angles, $\theta$ and $\phi$, respectively, and step vector s. The step vector is defined as $\mathbf{S}=\hat{\mathbf{c}} \times \hat{\mathbf{n}}$, where $\hat{\mathbf{c}}$ and $\hat{\mathbf{n}}$ are unit vectors normal to the C-plane and to the surface plane, respectively, so that $\mathbf{s}$ is parallel to the steps, descending to the right, and its modulus is the slope of the steps.

\section{Sapphire faceting}

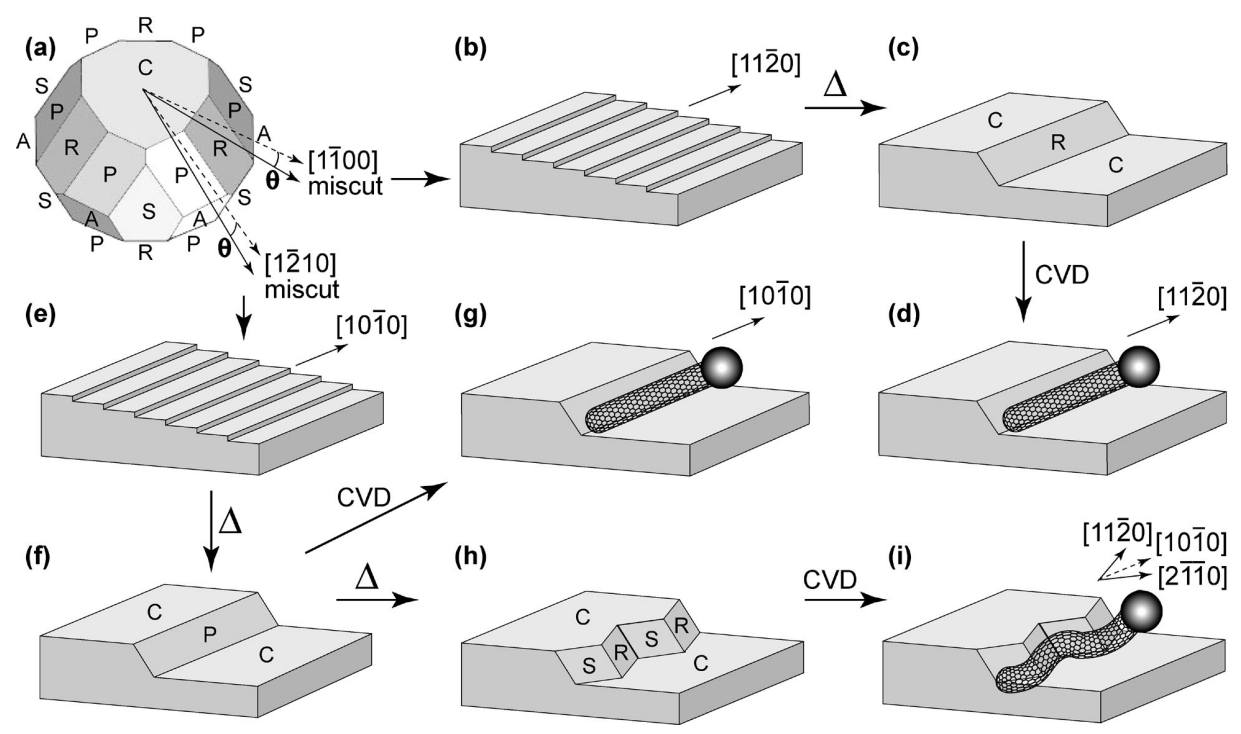


Figure S2. Possible morphologies of carbon nanotube graphoepitaxy obtained by miscut of C-plane sapphire, annealing, and CVD. (a) Equilibrium shape of $\alpha-\mathrm{Al}_{2} \mathrm{O}_{3}$, with facets $\mathrm{C}\{0001\}, \mathrm{R}\{1 \overline{1} 02\}$, $\mathrm{S}\{10 \overline{1} 1\}, \mathrm{P}\{11 \overline{2} 3\}$, and $\mathrm{A}\{11 \overline{2} 0\}$, in order of increasing surface energy. The same drawing is used to show the different miscut directions. (b) Miscut toward [1100] produces a vicinal $\alpha-\mathrm{Al}_{2} \mathrm{O}_{3}(0001)$

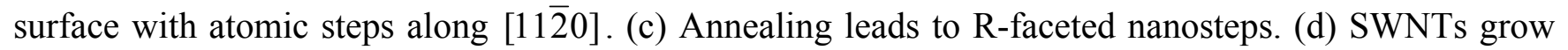
straight along [11 $\overline{2} 0]$ (the ball represents the catalyst nanoparticle). (e) Miscut toward [1 $\overline{2} 10]$ produces a vicinal $\alpha-\mathrm{Al}_{2} \mathrm{O}_{3}(0001)$ with atomic steps along[1010]. (f) Annealing initially leads to metastable Pfaceted nanosteps. (g) SWNTs grow straight along[1010]. (h) Further annealing from (f) leads to sawtooth-shaped S/R-faceted nanosteps. (i) SWNTs grow loosely conformal to the sawtooth nanosteps, with segments along $[11 \overline{2} 0]$ and $[2 \overline{1} \overline{1} 0]$.

\section{Methods}

C-plane sapphire wafers were purchased from Gavish Industrial \& Materials LTD., Omer, Israel (one side polished), with a miscut inclination angles of $4^{\circ}$ towards the [1100] direction. The material was received first as an ingot. The lattice orientation was identified by a back-reflection x-ray diffraction (XRD) method (see Figure $\mathrm{S} 3$ showing a XRD pattern from a $\alpha-\mathrm{Al}_{2} \mathrm{O}_{3}(0001)$ sample). After marking the exact directions, the ingots were sent back to Gavish Company for cutting according to the specifications described above, and mechanical polishing. The miscut inclination and azimuth angles were determined by X-ray diffraction, by an asymmetric double-exposure back-reflection method ${ }^{25}$ (see Figure S4a). The X-rays were produced by an Elliot GX6 rotating anode generator operating at $1.2 \mathrm{KW}$ and producing $\mathrm{Cu}$ radiation with a $200 \mu \mathrm{m}$ focus, with smallest wavelength of $0.41 \AA$ through $k_{\alpha}(1.54 \AA)$ and residual lower intensities of larger wavelengths. The sample, placed perpendicular to the X-ray beam, was first exposed to back reflections for $2 \mathrm{hrs}$, and then rotated by $180^{\circ}$ for a second exposure of 1 hr. In most cases a back reflection Laue pattern would have a larger number of reflections, which would be difficult to interpret. In order to reduce the number of spots and thus facilitate the recognition of both 
patterns, from the first and second exposures, a nickel filter was used to cut off the $k_{\beta} 1.39 \AA$ and shorter radiations. We know that $80 \%$ of the reflections are from radiation wavelengths between $1.5-1.8 \AA$. The miscut angle $\theta$ was measured by a systematic and an approximated method, both giving the same results: (i) The spots of the XRD patterns were placed on an appropriate Greninger chart in order to read the angular relations on the back reflection film, and then the spots were plotted on a stereographic projection to measure the miscut inclination and azimuth; (ii) The distance between the centers of the first and second patterns was measured and defined as $2 R$. Then the miscut inclinations given by $\theta=\tan ^{-1}(2 \mathrm{R} / \mathrm{L}) / 4$, where $L=3 \mathrm{~cm}$ is the distance between the sample and the X-ray sensitive film $(7 \mathrm{x} 7$ $\mathrm{cm}$ ) on which the Laue patterns were recorded. The result from such characterization is shown in Figure S4a. The green and red pattern represents the first, long, and second, short, exposures respectively. The miscut and azimuth angles where found to be $\theta=4.3 \pm 0.4^{\circ}$ and $\phi=2 \pm 5^{\circ}$ respectively. In addition, an independent AFM based method ${ }^{25}$ was utilized to characterize the miscut of the samples used in the present study. Figure S4b shows the AFM characterization of an annealed sample. The graph is a section analysis across the steps (red line in the inset), showing the step height, $H$, and the distance between the steps, $D$. Then the miscut can be calculated ${ }^{25}$ from the following relation $\theta=\sin ^{-1}(H / D)$. By doing a statistical analysis of the heights of 50 steps, the miscut angle was found to be $\theta=3.9 \pm 0.5^{\circ}$.

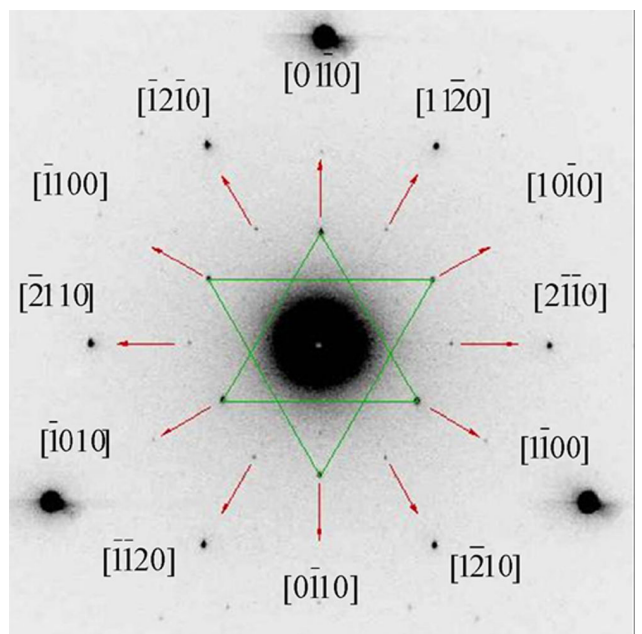

Figure S3. Back reflaction XRD pattern of a low-miscut $\alpha-\mathrm{Al}_{2} \mathrm{O}_{3}(0001)$ surface, showing the different lowindex lattice directions. 


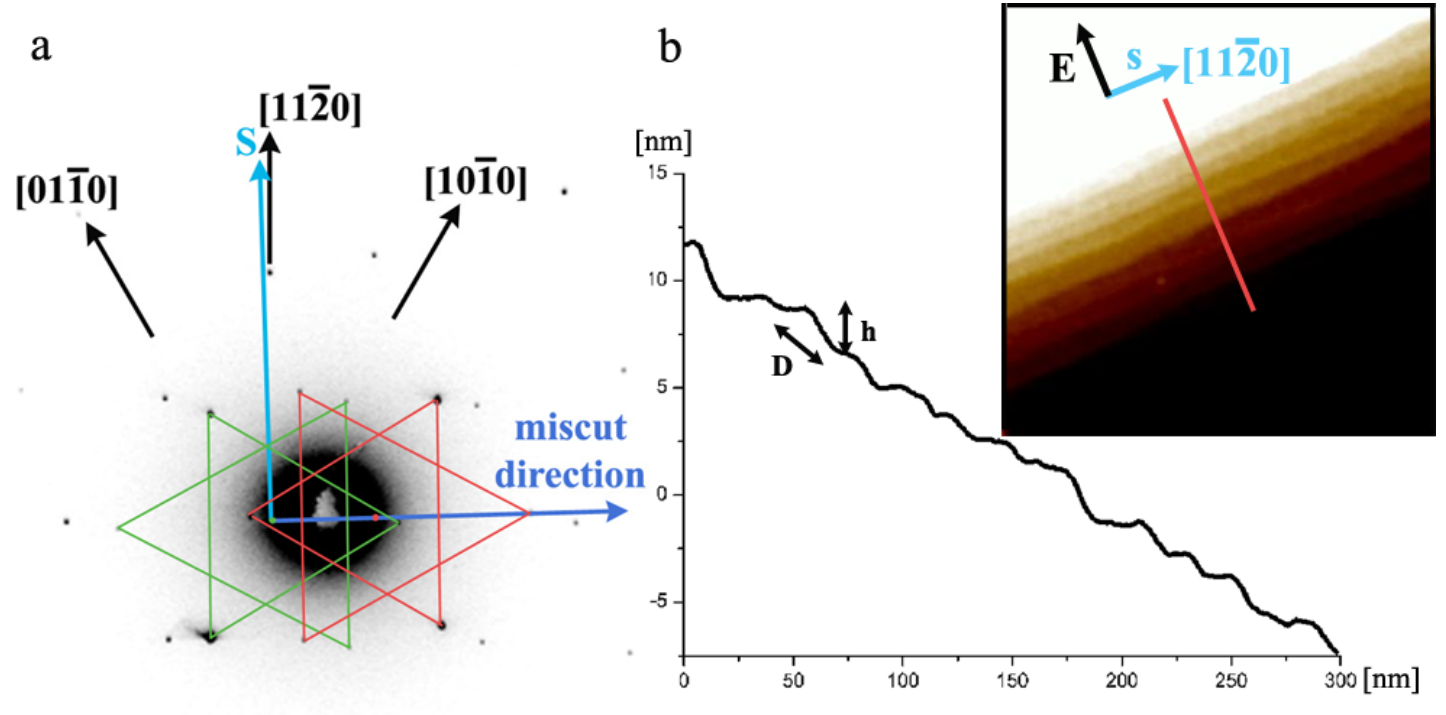

Figure S4. Characterization of the vicinal $\alpha-\mathrm{Al}_{2} \mathrm{O}_{3}$ (0001) surface. (a) Double-exposure backreflection XRD analysis showing the relevant lattice directions, step vector (s), and the miscut direction. The green and red patterns represent the first (long) and second (short) exposures respectively. It can be seen that the step vector is slightly deviated from the [1120] direction, with a miscut azimuth angle of $\phi$ $=2 \pm 5^{\circ}$. The miscut inclination angle is found to be $\theta=4.3 \pm 04^{\circ}$. (b) AFM characterization of the same surface after annealing $\left(1100^{\circ} \mathrm{C}\right.$ for $\left.10 \mathrm{hrs}\right)$. The staircase line represents a section analysis across the steps (red line in the inset AFM image). The spacing between the steps, $D$, and their height, $H$, are also shown for one step. By doing a statistical analysis on about 50 steps, the computed miscut angle is $\theta=\sin ^{-1}(H / D)=3.9 \pm 0.5^{\circ}$.

The first photolithographic step was carried out to deposit the Pt electrodes (photoresist Microposit S1805, Shipley), followed by electron beam evaporation (Edwards Auto 306) of $10 \mathrm{~nm}$ titanium (99.99\%, Holland Moran Ltd., Israel) and 90nm platinum (99.99\%, Holland Moran Ltd. Israel), while cooled with liquid nitrogen. The second photolithographic step was done in order to pattern the amorphous $\mathrm{SiO}_{2}$ islands or stripes. Then a thin layer, $10-20 \mathrm{~nm}$, of $\mathrm{SiO}_{2}(99.99 \%$, Kurt J. Lesker) was deposited by electron beam evaporation (Edwards Auto 306). The lithographic electrodes were 
connected with 4523AD Kulicke \& Soffa wire bonder, to external electrodes. A DC voltage of $50 \mathrm{~V}$ was applied between the electrodes (separation $25 \mu \mathrm{m}$ ) during CVD.

Single-wall carbon nanotubes (SWNTs) were grown by catalytic chemical vapor deposition (CVD), using ferritin protein as a precursor for monodisperse Fe nanoparticle catalyst, as previously reported $^{19,25,27}$. Substrates were first oxidized by oxygen plasma (March Plasmod GCM 200, 1-3 min, with $1 \mathrm{sccm}$ of $\mathrm{O}_{2}$ ), a ferritin solution of $0.1 \mathrm{~g} / \mathrm{L}$ (horse spleen ferritin, Sigma) was deposited on the samples for $10 \mathrm{~min}$, washed with deionizied water and blow dried, followed by a second plasma oxidation step, to eliminate all the organic matter. Finally, the CVD was carried out at $800^{\circ} \mathrm{C}$ for $10 \mathrm{~min}$ with a mixture of $60 \%$ Ar (99.998\%, Oxygen \& Argon Industries, Israel), 40\% $\mathrm{H}_{2}(99.999 \%$, Gordon Gas, Israel) and $0.2 \%$ ethylene (99.9\%, Gordon Gas, Israel) at $1 \mathrm{~atm}$ and flow rate of $1 \mathrm{~L} / \mathrm{min}$.

Atomic force microscopy (AFM) characterization was carried out in air tapping mode (Veeco, Multimode Nanoscope IV), using $70 \mathrm{kHz}$ etched Si probes (FESP, Nanoprobes).

Scanning Electron Microscopy (SEM), Supra 55VP FEG LEO, characterization was done in low working voltages, $0.5-5 \mathrm{kV}$.

\section{Additional images of SWNT crossbar arrays from different samples}
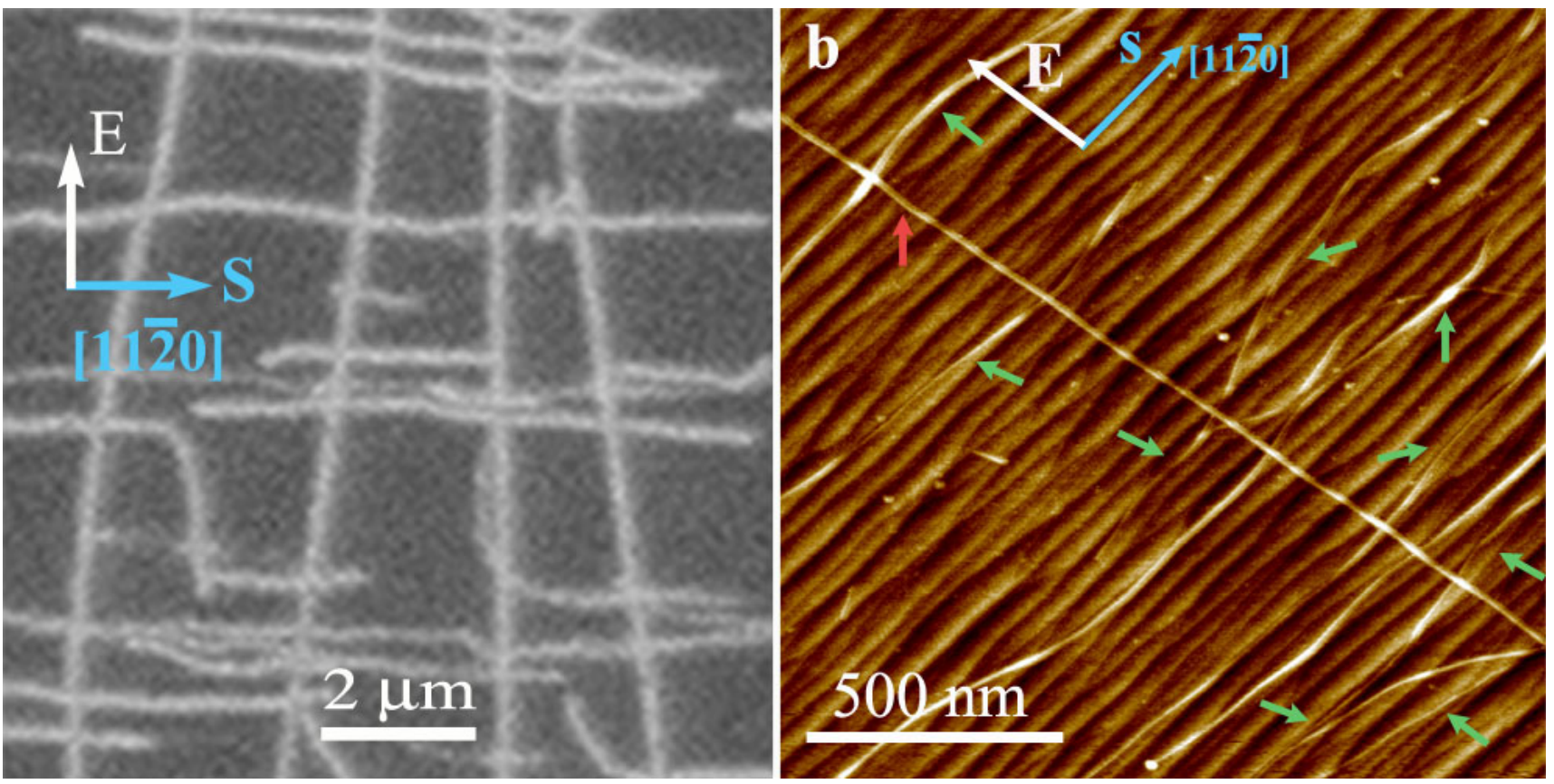
Figure S5. (a) SEM image showing a SWNTs grid structure produced by the orthogonal self-assembly process. (b) AFM topographic image in which a single nanotube aligned by the electric field (red arrow), crosses 9 graphoepitaxially aligned SWNTs (green arrows). Parts of the nanotubes can be seen hiding along the steps, which means that perhaps some nanotubes might be completely hidden by the stepped topography. 